\title{
Does Noninvasive Ventilation Delivery in the Ward Provide Early Effective Ventilation?
}

\author{
Carlo Olivieri MD, Luca Carenzo MD, Gian Luca Vignazia MD, Mauro Campanini MD, \\ Mario Pirisi MD, Francesco Della Corte MD, and Paolo Navalesi MD
}

\begin{abstract}
BACKGROUND: Although noninvasive ventilation (NIV) is increasingly used in general wards, limited information exists about its ability to provide effective ventilation in this setting. We aim to evaluate NIV delivered in the ward by assessing (1) overall time of application and occurrence of adverse events and (2) differences between daytime and nighttime NIV application. METHODS: We studied subjects with hypercapnic acute hypercapnic respiratory failure not fulfilling strict criteria for ICU admission, and excluded those who interrupted NIV prior to $48 \mathrm{~h}$. Time spent on NIV, presence and extent of air leaks, and occurrence of desaturations were assessed for the overall study period, and compared between daytime and nighttime. RESULTS: We enrolled 42 subjects, 25 of whom received NIV for at least $48 \mathrm{~h}$ and were included in the data analysis. NIV was successful for 20 subjects, who did not reach criteria for ICU admission. Both $\mathrm{PaCO}_{2}$ and $\mathbf{p H}$ significantly improved on average after $2 \mathrm{~h}$ and at the end of the study period. NIV was applied for $64.5 \%$ of the overall study period and had absent or compensated air leaks for $62.3 \%$ of the overall 48-h period. NIV was applied for $55.8 \%$ of daytime and for $79.3 \%$ of nighttime $(P<.01)$. Effective NIV application was significantly longer overnight $(76.9 \%)$ than during daytime $(53.2 \%)(P<.01)$. CONCLUSIONS: In selected subjects with hypercapnic acute respiratory failure not fulfilling criteria for ICU admission, the application of NIV in the ward is feasible; in addition, NIV can be safely administered overnight. Key words: noninvasive ventilation; acute respiratory failure; chronic obstructive pulmonary disease; exacerbation; intensive care unit; general ward; air-leaks. [Respir Care 2015;60(1):6-11. (C) 2015 Daedalus Enterprises]
\end{abstract}

\section{Introduction}

Early application of noninvasive ventilation (NIV) reduces the need for intubation and invasive ventilation and decreases mortality in patients with hypercapnic acute re-

Drs Olivieri, Vignazia, and Della Corte are affiliated with Anesthesia and Intensive Care, Maggiore della Carità University Hospital, Novara; Drs Carenzo, Pirisi, Della Corte and Navalesi are affiliated with the Department of Translational Medicine, Università del Piemonte Orientale Amedeo Avogadro, Novara; Drs Campanini and Pirisi are affiliated with Internal Medicine, Maggiore della Carità University Hospital, Novara; Dr Navalesi is affiliated with Anesthesia and Intensive Care, Sant'Andrea Hospital, Vercelli; and with CRRF Mons. L. Novarese, Moncrivello, Vercelli, Italy

Dr Olivieri and Dr Navalesi contributed to the development of a new interface (neither used nor mentioned in the present work), whose license for patent belongs to Intersurgical S.p.A., and receive royalties for that invention. Dr Navalesi's research laboratory has received equipment and spiratory failure secondary to an exacerbation of COPD..$^{1-3}$ In these patients, NIV is commonly applied intermittently, with an average daily application not exceeding, in general, 12 h. ${ }^{4}$ grants from Maquet Critical Care and Intersurgical S.p.A. Dr Navalesi also received honoraria/speaking fees from Maquet Critical Care, General Electric, Covidien AG, Hill-Rom, and GSK. Dr Pirisi is recipient of European FP7 grant 315322 for the project HCV Progress in Therapy with Novel Sialomimetic-Chimeric Inhibitors. The ResMed VPAP IV ventilators, the Mirage 4 face masks, the ResLink recording modules, and the ResView software were provided by ResMed.

Correspondence: Paolo Navalesi MD, Anestesia, Dipartimento di Medicina Traslazionale, Università del Piemonte Orientale Amedeo Avogadro Alessandria-Novara-Vercelli, Via Solaroli 17, 28100 Novara, Italy. E-mail: paolo.navalesi@med.unipmn.it.

DOI: $10.4187 /$ respcare. 03294 
NIV is increasingly used in general wards, especially in the face of ICU and high dependence unit (HDU) bed shortages. This earlier usage allows for safe application outside a more closely monitored setting. ${ }^{1,3,5-8}$ Compared with both ICU and HDU, general wards are characterized by reduced house staff availability, especially during the

\section{See the Related Editorial on Page 144}

night shift. The possibility of detecting NIV-related adverse events, such as air leaks, accidental disconnection, oxygen desaturation, and mask intolerance, is then arguably lower than in ICU and HDU, and adequate NIV application may not be guaranteed, especially overnight. Although these aspects may substantially affect effective ventilation and influence NIV outcome, ${ }^{9,10}$ surprisingly, so far no study has specifically evaluated them when NIV is delivered in the ward.

The aim of the present study was to evaluate subjects with hypercapnic ARF due to an exacerbation of COPD not requiring endotracheal intubation, NIV application in the medical ward during the first $48 \mathrm{~h}$ of treatment. Outcomes included time spent on NIV, occurrence and extent of air leaks, episodes of disconnection and desaturation, and the effects on arterial blood gases. In addition, we compared the effectiveness of daytime and nocturnal NIV.

\section{Methods}

The study was conducted at the University Hospital Maggiore della Carita in Novara, Italy, in accordance with the principles outlined in the Declaration of Helsinki. The local ethics committee approved the study protocol (CE $45 / 09$ protocol 222/CE det 118), and written informed consent was obtained from all study subjects.

\section{Study Subjects and Setting}

All adult patients admitted between March and December 2009 for hypercapnic ARF, defined as $\mathrm{pH}<7.35$ and $\mathrm{P}_{\mathrm{aCO}_{2}}>50 \mathrm{~mm} \mathrm{Hg}$, secondary to an exacerbation of COPD were screened for eligibility. All patients had known or likely COPD based on clinical history, physical examination, and chest $\mathrm{x}$-ray. Exclusion criteria were impairment of consciousness (Glasgow coma scale $<15$ ), vomit, ineffective cough, history of obstructive sleep apnea syndrome, pneumothorax, and any other cause indicating admission to ICU or HDU for either treatment and/or monitoring.

Predefined criteria for NIV failure were: (1) respiratory or cardiac arrest, (2) lack of improvement in arterial blood gases within $2 \mathrm{~h}$ after start of NIV application, (3) neurological deterioration, (4) inability to effectively clear secretions, (5)

\section{QUICK LOOK}

\section{Current knowledge}

Noninvasive ventilation (NIV) is increasingly used in general wards to allow earlier use, particularly with a shortage of ICU beds. The possibility of detecting NIVrelated adverse events, such as air leaks, accidental disconnection, oxygen desaturation, and mask intolerance, may be lower outside the ICU. The use of NIV in general wards remains controversial.

\section{What this paper contributes to our knowledge}

NIV delivered in a general ward, supervised by an ICUdedicated team was feasible for the first $48 \mathrm{~h}$ in selected subjects with hypercapnic respiratory failure and exacerbations of COPD. NIV was delivered in the ward without an increase in overnight complications.

life-threatening arrhythmias, or (6) hypotension (ie, systolic arterial pressure $<90 \mathrm{~mm} \mathrm{Hg}$ nonresponsive to fluids and/or requiring infusion of inotropic or vasoactive drugs). Subjects with interrupted NIV treatment because of NIV failure, recovery from hypercapnic ARF, or for other reasons within the first $48 \mathrm{~h}$ were excluded from the data analysis.

NIV was applied through an oronasal mask (Mirage 4, ResMed, Bella Vista, Australia) using a bi-level ventilator (ResMed VPAP IV, ResMed) set in spontaneous/timed mode. The size of the mask was chosen to guarantee the best comfort while minimizing air leaks. Expiratory positive airway pressure was set between 4 and $8 \mathrm{~cm} \mathrm{H}_{2} \mathrm{O}$ according to subject's comfort and triggering performance; inspiratory positive airway pressure was initially set at $8 \mathrm{~cm} \mathrm{H}_{2} \mathrm{O}$ above expiratory positive airway pressure and then increased by steps of $2 \mathrm{~cm} \mathrm{H}_{2} \mathrm{O}$ to the highest tolerated level not generating air leaks. The maximum limit of the total applied pressure was $25 \mathrm{~cm} \mathrm{H}_{2} \mathrm{O}$. Ventilator backup rate was set at 8 breaths/min with an inspiratory time of $1.2 \mathrm{~s}$. Triggering and cycling-off were set at the discretion of the attending physician to maximize subject comfort and avoid visible subject-ventilator asynchrony. Oxygen was supplemented via a low-pressure inlet and titrated to achieve a $\mathrm{S}_{\mathrm{pO}_{2}}$ between $90 \%$ and $94 \%$. The ventilator has a built-in sound alarm for disconnections. An additional external device was utilized for monitoring and alarming heart rate and $\mathrm{S}_{\mathrm{pO}_{2}}$.

The ventilator was equipped with a dedicated recording module (ResLink, ResMed), whose accuracy has been previously validated. ${ }^{11}$ ResLink continuously records air flow, airway pressure, and breathing frequency; it also records $\mathrm{S}_{\mathrm{pO}_{2}}$ and heart rate via a connected pulse oximeter. Data were then downloaded and off-line analyzed on a personal computer using a dedicated software (ResView, ResMed). 
All subjects started NIV in the emergency department in association with the standard medical therapy, which included oxygen, antibiotics, steroids, and inhaled bronchodilators. ${ }^{12}$ Subjects were carefully monitored and attended by the ICU staff in a protected environment within the emergency department for the first $2 \mathrm{~h}$ after NIV initiation. Arterial blood was then sampled for gas analysis, and, when the subject improved according to predefined criteria, she/he was transferred to the ward irrespective of the time of the day. A dedicated ICU team was responsible for NIV implementation, while the medical and nursing staff of the ward provided daily clinical assistance. Sedatives were never administered. Subjects were evaluated at least 2 times daily by the ICU team and any further time it was considered necessary by the ward staff. All physicians and nurses of the wards had previously attended a dedicated training program including theoretical and practical modules.

\section{Protocol and Measurements}

All subjects received a prescription to receive NIV "as much as possible" throughout the $48 \mathrm{~h}$ of observation. We considered the epoch between $7 \mathrm{AM}$ and 10 PM as daytime, and the remaining period between $10 \mathrm{PM}$ and $7 \mathrm{AM}$ as nighttime. ${ }^{13}$ During daytime, the subject-to-nurse ratio was 15:2; during nighttime, it was 15:1. The actual time of NIV application was computed and expressed as percentage of the overall 48-h study period, and, separately, of daytime and nighttime. The actual time of NIV application was divided according to the occurrence of air leaks as follow: (1) absent, (2) compensated, that is, $\leq 24 \mathrm{~L} / \mathrm{min}$, corresponding to the ventilator capacity for compensation, as indicated by the manufacturer, (3) uncompensated ( $>24$ and $<80 \mathrm{~L} / \mathrm{min}$ ), and (4) disconnection, defined as air leak $\geq 80 \mathrm{~L} / \mathrm{min}$ for more than $30 \mathrm{~s}$. We considered NIV effective when air leaks were absent or compensated. Oxygen desaturations, defined as reduction of $\mathrm{S}_{\mathrm{pO}_{2}}$ greater than $4 \%$ from baseline, were also assessed. ${ }^{14} \mathrm{We}$ considered relevant the differences in the time of NIV application between day and night when $\geq 10 \%$.

Arterial blood was sampled for gas analysis before starting NIV, $2 \mathrm{~h}$ after NIV initiation, and at least once daily thereafter or whenever clinically warranted. After the 48-h study period, NIV was continued for as long as clinically required.

\section{Statistical Analysis}

Normal distribution of data were tested through the Kolmogorov-Smirnov analysis. Data are accordingly presented as mean \pm standard deviation or median [interquartile]. Head-to-head comparisons between daytime and nighttime variables were analyzed by two tailed unpaired $t$ test or
Table 1. Subject Characteristics at Enrollment

\begin{tabular}{lc}
\hline \hline Subjects $(N)$ & 25 \\
Age $(y)$ & $79(67-83)$ \\
Female, $n(\%)$ & $14(56)$ \\
APACHE II on study entry & $17(12-20)$ \\
EPAP at NIV start $\left(\mathrm{cm} \mathrm{H}_{2} \mathrm{O}\right)$ & $7(5-8)$ \\
IPAP at NIV start $\left(\mathrm{cm} \mathrm{H}_{2} \mathrm{O}\right)$ & $15(13-18)$ \\
& \\
\hline All values, except gender, are expressed as median and 25 th to 75 th percentile. \\
APACHE = acute physiology and chronic health evaluation \\
EPAP = expiratory positive airway pressure \\
IPAP $=$ inspiratory positive airway pressure \\
NIV = noninvasive ventilation \\
\hline
\end{tabular}

Mann-Whitney test, as indicated. Blood gas values were analyzed with the one-way analysis of variance for repeated measures. Differences in the proportion of daytime and nighttime spent with effective NIV were analyzed using the chi-square test. We considered statistically significant $P$ values $<.05$.

\section{Results}

We enrolled 42 subjects. Seventeen subjects did not complete the 48-h study period and were excluded from the analysis. Thirteen subjects recovered and interrupted NIV before $48 \mathrm{~h}$, and 4 subjects met NIV failure criteria. Of this latter subgroup of subjects, 1 interrupted NIV for intolerance and was then treated in the ward with standard therapy only, while 3 deteriorated and either were transferred to ICU and intubated ( 2 subjects), or remained in the ward to receive palliative care (1 subject). Accordingly, we included in the data analysis 25 subjects, whose group mean characteristics are shown in Table 1. Of these 25 subjects, $52 \%$ had associated chronic heart failure, $36 \%$ hypertension, and $12 \%$ peripheral vascular disease. Noninsulin-dependent diabetes was present in $28 \%$ of the subjects, whereas chronic renal failure and morbid obesity were both observed in 4 subjects $(16 \%)$. The rate of NIV failure was $21 \%$ for the overall 42 subjects enrolled in the study and $20 \%$ for the 25 subjects included in the data analysis. After the study period, 1 subject was transferred to ICU and intubated, while 4 refused intubation and died in the ward.

Arterial blood gases at study enrollment $\left(\mathrm{t}_{0}\right)$, after $2 \mathrm{~h}$ $\left(\mathrm{t}_{2}\right)$, and after $48 \mathrm{~h}\left(\mathrm{t}_{48}\right)$ are presented in Table 2. Compared with $\mathrm{t}_{0}$, both $\mathrm{P}_{\mathrm{aCO}}$ and $\mathrm{pH}$ significantly improved at $\mathrm{t}_{2}$ and $\mathrm{t}_{48}$, whereas the increase in $\mathrm{P}_{\mathrm{aO}_{2}}$ did not achieve statistical significance.

Overall, NIV was applied for $775 \mathrm{~h}$ and $33 \mathrm{~min}$, corresponding to $64.5 \%$ of the $1,200 \mathrm{~h}$ of total time (ie, $48 \mathrm{~h} \times$ 25 subjects). The average time of NIV application per subject was $31 \pm 8 \mathrm{~h}$. NIV was interrupted because of nursing, therapeutic or diagnostic procedures, meals, and 
Table 2. Arterial Blood Gas Values on Study Entry, $2 \mathrm{~h}$, and $48 \mathrm{~h}$ After Start of NIV

\begin{tabular}{lcccccc}
\hline \hline & $\mathrm{t}_{0}$ & $\mathrm{t}_{2}$ & $\mathrm{t}_{48}$ & \multicolumn{2}{c}{$P$} \\
\cline { 5 - 7 } & $(n=25)$ & $(n=25)$ & $(n=25)$ & $\mathrm{t}_{0} \mathrm{vs} \mathrm{t}_{2}$ & $\mathrm{t}_{0} \mathrm{vs} \mathrm{t}_{48}$ \\
\hline $\mathrm{pH}$ & $7.26 \pm 0.06$ & $7.35 \pm 0.07$ & $7.40 \pm 0.05$ & $<.001$ & $<.0001$ \\
$\mathrm{P}_{\mathrm{aCO}_{2}}$ & $72.3 \pm 14.9$ & $58.9 \pm 13.6$ & $53.6 \pm 13.3$ & $<.01$ & $<.0001$ \\
$(\mathrm{~mm} \mathrm{Hg})$ & & & & & \\
$\mathrm{P}_{\mathrm{aO}_{2}}^{(\mathrm{mm} \mathrm{Hg})}$ & $68.9 \pm 25.0$ & $74.1 \pm 20.5$ & $80.3 \pm 34.5$ & .42 & .26 \\
& & & & &
\end{tabular}

Values are expressed as mean $\pm \mathrm{SD}$

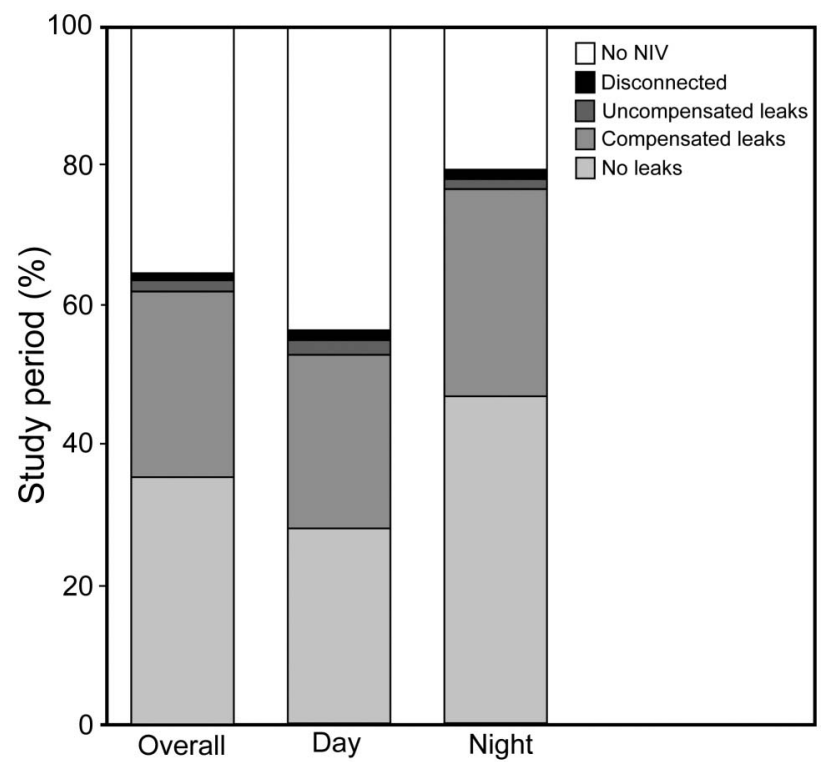

Fig. 1. Percent of the study period spent with no air leaks, compensated leaks (ie, $\leq 24 \mathrm{~L} / \mathrm{min}$ ), uncompensated leaks (ie, leak 24-80 $\mathrm{L} / \mathrm{min}$ ), and with the subject disconnected (ie, leak $\geq 80 \mathrm{~L} / \mathrm{min}$ for $>30 \mathrm{~s}$ ), during NIV.

subject's request. Effective NIV was applied for $62.3 \%$ of the 48-h study period. As depicted in Figure 1, the proportion of the study period during which air leaks were absent or negligible was $35.6 \%$; compensated leaks accounted for $26.7 \%$ of the study period, and uncompensated leaks and complete disconnection for $1.8 \%$ and $0.4 \%$, respectively. During the remaining $35.6 \%$ of the time, subjects did not receive NIV at all. The average values of $\mathrm{S}_{\mathrm{pO}_{2}}$, heart rate, and breathing frequency for the overall 48-h period are shown in Table 3. Air leaks extent, rate and duration of disconnections, and number of oxygen desaturations associated with disconnections during the 48-h of study period are also presented in Table 3.

NIV was applied for $55.8 \%$ of daytime and for $79.3 \%$ of nighttime $(P<.01)$, as depicted in Figure 1. Individual rates of NIV application during daytime (left) and nighttime (right) are shown in Figure 2. In 7 subjects, the dif-
Table 3. Physiologic Parameters, Amount of Air Leaks, Number and Duration of Disconnections, and Number of Disconnection-Associated Desaturations

\begin{tabular}{lcccc}
\hline \hline & \multicolumn{1}{c}{ Overall } & Day & Night & $P$ \\
\hline $\mathrm{S}_{\mathrm{pO}_{2}}(\%)$ & $91.6 \pm 4.7$ & $92.2 \pm 4.6$ & $91.0 \pm 4.6$ & .19 \\
Heart rate (beats/min) & $80.1 \pm 18.8$ & $82.0 \pm 19.3$ & $78.0 \pm 18.1$ & .76 \\
Breathing frequency & $23.5 \pm 9.8$ & $23.4 \pm 9.7$ & $23.7 \pm 9.8$ & .98 \\
$\quad$ (breaths/min) & & & & \\
Air leaks (L/min) & $8.3 \pm 15.3$ & $10.2 \pm 17.6$ & $6.4 \pm 12.2$ & .08 \\
Disconnections/h $(n)$ & $0.40 \pm 0.44$ & $0.24 \pm 0.26$ & $0.16 \pm 0.30$ & .10 \\
$\begin{array}{c}\text { Disconnection duration } \\
\quad(\text { min) }\end{array}$ & $1.1 \pm 1.3$ & $1.2 \pm 1.1$ & $1.0 \pm 1.3$ & .26 \\
$\begin{array}{c}\text { Disconnection-associated } \\
\quad \text { desaturations/h }(n)\end{array}$ & $0.21 \pm 0.24$ & $0.15 \pm 0.21$ & $0.06 \pm 0.09$ & .06 \\
& & & &
\end{tabular}

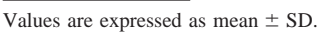

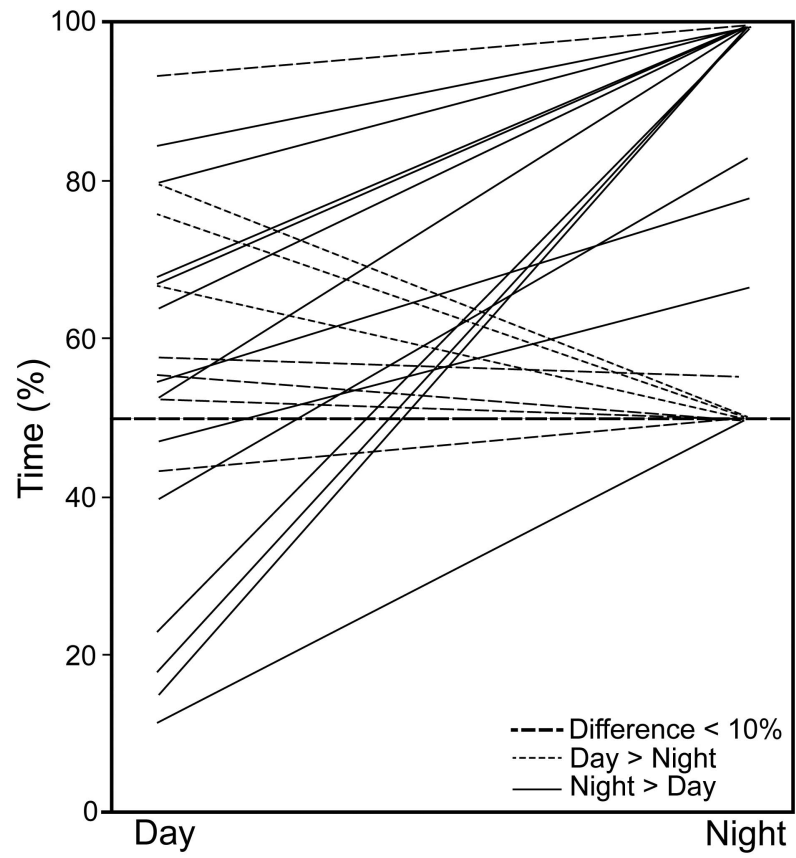

Fig. 2. Percentages of time of NIV application during daytime and nighttime are shown for each subject. The horizontal dashed line indicates $50 \%$ of the overall time.

ference in the rate of NIV application between day and night was $<10 \%$ and therefore considered unimportant; in 14 of the remaining 18 subjects, NIV was applied for higher percentages of time overnight than during daytime $(P<.01)$. Moreover, the rate of time during which NIV was effectively delivered was significantly greater overnight than during daytime ( $76.9 \%$ and $53.2 \%$, respectively, $P<.01$ ) (Fig. 1). Noteworthy, also shown in Figure 1, the rate of time without air leaks at all was higher overnight than during daytime $(46.8 \%$ and $28.0 \%$, respectively, $P<.01$ ), while there were no significant differences be- 
tween day and night with respect to the time spent with compensated air leaks $(25.2 \%$ and $30.1 \%$, respectively, $P=.75)$, uncompensated air leaks $(2.1 \%$ and $1.9 \%$, respectively, $P=.99)$, and disconnections $(0.5 \%$ and $0.5 \%$, respectively, $P=0.99$ ). As displayed in Table 3 , the average values of $\mathrm{S}_{\mathrm{pO}_{2}}$, heart rate, and breathing frequency were not significantly different between daytime and nighttime; extent of air leaks, rate and duration of disconnections, and number of desaturations associated with disconnections were also not different between day and night.

\section{Discussion}

The major findings of our study are: (1) in subjects with hypercapnic ARF secondary to an exacerbation of COPD who do not meet strict criteria for ICU or HDU admission, NIV provides effective ventilation in medical wards with trained nursing and medical staff, at least for the initial $48 \mathrm{~h}$ of the acute phase; (2) NIV adverse events were relatively few and of minor clinical importance; and (3) the amount of time that NIV was effectively applied was greater overnight than during the daytime, with air leaks, disconnections, and desaturations not different between day and night.

Although first proposed years ago, ${ }^{3,6,7}$ the use of NIV outside the ICU has increased, in particular in those countries where tight spending reviews reduce healthcare resources. ${ }^{15}$ Because these constraints do not exclude ICU services, physicians often face the dilemma of guaranteeing proper treatments at contained costs. ${ }^{16}$ Under this perspective, delivering NIV outside the ICU can prevent withholding a beneficial intervention because of scarcity of resources. Ideally, NIV should be applied in a monitored setting with low patient-to-nurse ratio, ${ }^{17}$ but in the real world, the shortage of ICU and HDU beds may lead to postponing or denying NIV application with potential detrimental effects, such as further deterioration, reduced chance of successful response to NIV, and therefore worsening of patient's outcome. ${ }^{4,18,19}$

Guidelines advise NIV application for an exacerbation of COPD with $\mathrm{pH}<7.35,{ }^{10}$ and recommend ICU or HDU admission when $\mathrm{pH}$ is $<7.25$ or worsening despite NIV. ${ }^{11}$ Due to shortage of ICU bed provision and lack of HDU in our hospital, we adopt less restrictive criteria for NIV application in the ward. Carlucci et $\mathrm{a}^{20}$ showed that the clinical practice for NIV in an exacerbation of COPD subjects with hypercapnic ARF changes over time, more severely ill patients being progressively treated with reduced risk of failure, and that staff training and increased knowledge of the technique enhance the ability to treat patients outside the ICU. In our center, NIV has been used for several years in the wards, where nursing and medical staff undergo formal NIV educational courses and training on a regular basis. In addition, the subjects included in the study had arterial blood gases evaluated within $2 \mathrm{~h}$ from NIV initiation, and only those who improved and had intact sensorium and stable cardiovascular conditions were treated in the ward. Our subjects had an average $\mathrm{pH}$ on study entry of $7.26 \pm 0.06$ and showed a $20 \%$ rate of NIV failure, a value slightly higher than the $15 \%$ reported by Plant et $\mathrm{al}^{3}$ in patients with an average $\mathrm{pH}$ of 7.31 , but lower than the failure rate of $36 \%$ observed in the subgroup of patients with $\mathrm{pH}<7.30$.

The extent of uncompensated air leaks in our study is definitely lower than previously reported. ${ }^{9}$ This finding may be due to the improvement achieved by newer interfaces, and to the specific practical training in interface management that was part of the educational program provided to the nurses. Somewhat surprisingly, considering the reduced staff availability, NIV was effectively administered for longer portions of time overnight, compared with daytime, without increased adverse events such as excessive air leaks, disconnections and desaturations. Furthermore, as indicated in Figure 2, all subjects received NIV for at least $50 \%$ of nighttime. To our knowledge, no study has so far evaluated this aspect. Although we do not have a clear interpretation for these findings, we believe that the reduced need for environmental interaction overnight could be a partial explanation. Nevertheless, our data suggest that, in subjects who are not ventilator-dependent and require treatment only for part of the day, a large fraction of the time committed to NIV application can be covered by the night hours.

Our study has limitations deserving discussion. First, it is a single-center uncontrolled cohort study, with a limited number of subjects, performed in an institution where NIV is delivered by experienced ICU physicians and well-trained ward personnel. It would therefore be meaningless attempting to draw conclusions on the clinical outcome of our subjects and incorrect to consider our results extendable to any center, irrespective of the level of expertise and training. Second, to assess differences in effective ventilation between daytime and nighttime, we divided each full day in two epochs, daytime (from 7 AM to $10 \mathrm{PM}$ ) and nighttime (from 10 PM to $7 \mathrm{AM}$ ) and arbitrarily considered 2 full days necessary to provide valuable information on this aspect. As we included in the data analysis only the subjects who received NIV for at least $48 \mathrm{~h}$, we likely excluded those with the mildest (ie, early recovery) or most severe (ie, NIV failure) hypercapnic ARF. Third, because we used the same equipment (oronasal mask and ventilator) for all the subjects, it could be argued that our results may not be extended to other setup. We used this specific setup for two reasons: first, the ventilator utilized was equipped with a dedicated recording module, easy to use and previously validated; ${ }^{12}$ second, the use of a standardized setup facilitated the educational and training process. Fourth, subjects did not receive NIV for about $37 \%$ of the time. 
Because our study was not designed for this purpose, nonetheless, we did not log the events determining NIV interruptions. Fifth, we did not perform sleep studies in our subjects. Sleep disturbances during NIV have been described, ${ }^{21,22}$ and early sleep abnormalities have been associated with late ( $\geq 48 \mathrm{~h}$ ) NIV failure..$^{22}$ It is worth noting that we excluded patients with a history of obstructive sleep apnea syndrome; in addition, because sleep interferences caused by noise, light, and staff activities are definitely lower in the ward, as opposed to both ICU and HDU, the risk of sleep fragmentation should accordingly be reduced.

\section{Conclusions}

Ward-based NIV supervised by an ICU dedicated team is feasible for selected patients with hypercapnic ARF secondary to COPD without strict criteria for treatment and/or monitoring in ICU or HDU. In fact, we found that most of the time was spent with air leaks absent or adequately compensated. In addition, NIV can be safely administered overnight, with no additional risk compared with daytime, of ineffective ventilation.

\section{REFERENCES}

1. Nava S, Hill N. Non-invasive ventilation in acute respiratory failure. Lancet 2009;374(9685):250-259.

2. Lightowler JV, Wedzicha JA, Elliott MW, Ram FS. Non-invasive positive pressure ventilation to treat respiratory failure resulting from exacerbations of chronic obstructive pulmonary disease: Cochrane systematic review and meta-analysis. BMJ 2003;326(7382):185.

3. Plant PK, Owen JL, Elliott MW. Early use of non-invasive ventilation for acute exacerbations of chronic obstructive pulmonary disease on general respiratory wards: a multicentre randomised controlled trial. Lancet 2000;355(9219):1931-1935.

4. Nava S, Navalesi P, Conti G. Time of non-invasive ventilation. Intensive Care Med 2006;32(3):361-370.

5. Collaborative Research Group of Noninvasive Mechanical Ventilation for Chronic Obstructive Pulmonary Disease. Early use of noninvasive positive pressure ventilation for acute exacerbations of chronic obstructive pulmonary disease: a multicentre randomized controlled trial. Chin Med J (Engl) 2005;118(24):2034-2040.

6. Cabrini L, Idone C, Colombo S, Monti G, Bergonzi PC, Landoni G, et al. Medical emergency team and non-invasive ventilation outside ICU for acute respiratory failure. Intensive Care Med 2009;35(2): 339-343.
7. Chiumello D, Conti G, Foti G, Giacomini MM, Braschi A, Iapichino G. Non-invasive ventilation outside the intensive care unit for acute respiratory failure. Minerva Anestesiol 2009;75(7-8):459-466.

8. Khalid I, Sherbini N, Qushmaq I, Qabajah MR, Nisar A, Khalid TJ, Hamad WJ. Outcome of patients treated with noninvasive ventilation by medical emergency team on the wards. Respir Care 2014;59(5): e89-e90.

9. Carlucci A, Richard JC, Wysocki M, Lepage E, Brochard L. Noninvasive versus conventional mechanical ventilation: an epidemiologic survey. Am J Respir Crit Care Med 2001;163(4):874-880.

10. Global Initiative for Chronic Obstructive Lung Disease. Global strategy for the diagnosis, management and prevention of COPD, Updated 2013. Available from: http://www.goldcopd.org/Guidelines/ guidelines-resources.html. Accessed September 24, 2014

11. Rabec C, Georges M, Kabeya NK, Baudouin N, Massin F, ReybetDegat O, Camus P. Evaluating noninvasive ventilation using a monitoring system coupled to a ventilator: a bench-to-bedside study. Eur Respir J 2009;34(4):902-913.

12. Demoule A, Girou E, Richard JC, Taillé S, Brochard L. Increased use of noninvasive ventilation in French intensive care units. Intensive Care Med 2006;32(11):1747-1755.

13. Needleman J, Buerhaus P, Mattke S, Stewart M, Zelevinsky K. Nurse-staffing levels and the quality of care in hospitals. N Engl J Med 2002;346(22):1715-1722.

14. Wynne JW, Block AJ, Hemenway J, Hunt LA, Flick MR. Disordered breathing and oxygen desaturation during sleep in patients with chronic obstructive lung disease (COLD). Am J Med 1979;66(4): 573-579.

15. Karanikolos M, Mladovsky P, Cylus J, Thomson S, Basu S, Stuckler $\mathrm{D}$, et al. Financial crisis, austerity, and health in Europe. Lancet 2013;381(9874):1323-1331.

16. Truog RD, Brock DW, Cook DJ, Danis M, Luce JM, Rubenfeld GD, et al. Rationing in the intensive care unit. Crit Care Med 2006;34(4): 958-963.

17. Elliott MW, Confalonieri M, Nava S. Where to perform noninvasive ventilation? Eur Respir J 2002;19(6):1159-1166.

18. Cabrini L, Antonelli M, Savoia G, Landriscina M. Non-invasive ventilation outside of the intensive care unit: an Italian survey. Minerva Anestesiol 2011;77(3):313-322.

19. Squadrone E, Frigerio P, Fogliati C, Gregoretti C, Conti G, Antonelli $\mathrm{M}$, Costa R, et al. Noninvasive vs invasive ventilation in COPD patients with severe acute respiratory failure deemed to require ventilatory assistance. Intensive Care Med 2004;30(7):1303-1310.

20. Carlucci A, Delmastro M, Rubini F, Fracchia C, Nava S. Changes in the practice of non-invasive ventilation in treating COPD patients over 8 years. Intensive Care Med 2003;29(3):419-425.

21. Carenzo L, Olivieri C, Vignazia GL, Navalesi P. Idiopathic CheyneStokes respiration in acute respiratory acidosis. Am J Respir Crit Care Med 2011;184(11):1313.

22. Roche Campo F, Drouot X, Thille AW, Galia F, Cabello B, d'Ortho MP, Brochard L. Poor sleep quality is associated with late noninvasive ventilation failure in patients with acute hypercapnic respiratory failure. Crit Care Med 2010;38(2):477-485.

This article is approved for Continuing Respiratory Care Education credit. For information and to obtain your CRCE

(free to AARC members) visit

www.rcjournal.com

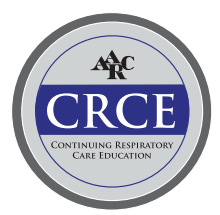

